\title{
$O$ uso da medida canadense de desempenho ocupacional (COPM) em saúde mental*
}

\author{
The use of the canadian occupational \\ performance measure in mental health
}

\author{
Simone Costa de Almeida Bastos ${ }^{1}$, Marisa Cotta Mancini², \\ Rúbia Marques Pyló ${ }^{3}$
}

\begin{abstract}
BASTOS, S. C. A.; MANCINI, M. C.; PYLÓ, R. M. O uso da medida canadense de desempenho ocupacional (COPM) em saúde mental. Rev. Ter. Ocup. Univ. São Paulo, v. 21, n. 2, p. 104-110, maio/ago. 2010.

RESUMO: A COPM é em um instrumento capaz de mensurar o impacto de uma intervenção para um indivíduo, tendo como finalidade detectar mudanças na percepção do cliente sobre seu desempenho ocupacional ao longo do tempo, bem como mudanças em sua satisfação em relação a esse desempenho. Realizou-se uma bibliografia comentada, analisando-se os artigos publicados nos últimos sete anos sobre a utilização da COPM no campo da saúde mental. Objetivou-se verificar a área de desempenho ocupacional em que se concentram as dificuldades identificadas pelos clientes da saúde mental, bem como as vantagens e limitações da utilização do instrumento com esse público. A pesquisa foi realizada nas bases de dados eletrônicas Medline, OTseeker e CINAHL, no período de 2002-2009. Os resultados sugerem que as condições de saúde na área de saúde mental repercutem em dificuldades de desempenho ocupacional nas três áreas. Convém destacar a singularidade das informações fornecidas pelo uso da COPM no que diz respeito ao desempenho ocupacional do cliente, bem como o (re) conhecimento que o uso dessa medida propicia sobre a atuação do terapeuta ocupacional na equipe multidisciplinar. A despeito das dificuldades existentes na aplicação da COPM, pode-se afirmar que esse instrumento foi identificado como uma ferramenta útil para uso na saúde mental.
\end{abstract}

DESCRITORES: Avaliação em saúde/métodos; Indicadores; Avaliação de recursos humanos em saúde; Saúde mental; Medidas; Terapia ocupacional.

\footnotetext{
* Trabalho apresentado como pôster no XV Congresso Brasileiro de Terapia Ocupacional, Fortaleza, 12 a 16 de outubro de 2009.

1. Professora assistente do Departamento de Terapia Ocupacional da UFMG, Mestre em Sociologia pela UFMG.

2. Professora titular do Departamento de Terapia Ocupacional da UFMG, Pós-doutora no Centro de Estudos ecológicos em percepção e ação - CESPA da Universidade de Connecticut.

3. Terapeuta Ocupacional.

Endereço para correspondência: salmeida@ufmg.br
} 


\section{INTRODUÇÃO}

A s mudanças em curso no cenário sócioeconômico mundial têm repercutido na atuação dos profissionais da área da saúde, acarretando a necessidade de maior sistematização de sua prática. Essa nova realidade profissional configurou-se a partir de cortes no orçamento, crescente aumento dos seguros de saúde, sistemas de pagamento pautados na definição de objetivos terapêuticos, relação custo-efetividade da intervenção, aumento no número de consumidores de saúde e na disponibilidade da informação que os consumidores têm sobre a doença e as terapêuticas (SAMPAIO et al., 2002).

Especificamente em relação ao campo da reabilitação, essas profissões emergiram durante a década de 1980, saindo da situação de um relativo desconhecimento para se tornarem essenciais à rede de cuidados em saúde. Atualmente, a disponibilização de serviços de reabilitação visando ao aumento da capacidade funcional de clientes com severas limitações físicas e/ou cognitivas dá credibilidade ao sistema de saúde (DEJONG; SUTTON, 1995). Diante dessa conjuntura, a demonstração de resultados dos procedimentos é decisiva para o marketing, preço e avaliação de um serviço de reabilitação. Consequentemente, o uso de medidas mais objetivas e psicometricamente mais rigorosas para informar sobre o estado e evolução do cliente durante o processo terapêutico adquiriu particular importância (SAMPAIO et al., 2002).

A Medida Canadense de Desempenho Ocupacional (COPM) é uma medida de resultados, ou seja, um instrumento capaz de mensurar o impacto de uma intervenção para um indivíduo (POLLLOCK et al., 2003). Trata-se de um teste padronizado, já que requer métodos para administração e pontuação (EYSSEN et al., 2006).

A COPM é administrada por meio de uma entrevista semi-estruturada e, devido a esse formato, permite que os indivíduos identifiquem qualquer atividade de importância que consideram de difícil execução num contexto de adoecimento (DEDDING et al., 2004; EYSSEN et al., 2006). Foi construída para ser utilizada por terapeutas ocupacionais, tendo como finalidade mensurar mudanças na percepção do cliente sobre seu desempenho ocupacional ao longo do tempo, bem como mudanças em sua satisfação com esse desempenho (CUP et al., 2003; EYSSEN et al., 2006). Esse teste foca os problemas e necessidades dos clientes de forma individual e não é específico para determinada condição de saúde, sendo utilizado para estabelecer objetivos, planejar o tratamento e mensurar o progresso do cliente (CUP et al., 2003; CARSWELL, 2004; DEDDING et al., 2004).

A COPM é baseada no Modelo Canadense de Desempenho Ocupacional, que incorpora os princípios da
Prática Centrada no Cliente. Nesse modelo de prática, o teste pode ser envolvido em todas as fases do tratamento: na definição das prioridades, na decisão de estratégias adequadas para a intervenção e na documentação de mudanças resultantes do tratamento (DEDDING et al., 2004). Pesquisas demonstram que a prática centrada no cliente está associada à maior satisfação do cliente e adesão aos serviços de saúde, comparada com outros modelos de prática (DEDDING et al., 2004; WRESSLE et al., 2002). Evidências revelam ainda que, quando a escolha do cliente e a auto-avaliação são incorporadas ao tratamento, há aumento da motivação e participação do cliente favorecendo sua recuperação funcional (CUP et al., 2003).

Por meio da COPM, o cliente seleciona as atividades de que precisa e deseja realizar, ou seleciona aquelas que se espera que esse realize, mas que não tem conseguido ou não está satisfeito com seu desempenho. Essas atividades podem enquadrar-se em qualquer uma das áreas de desempenho: auto-cuidado, trabalho e lazer. O cliente então atribui a cada atividade uma importância, variando essa de 1 (não é importante de nenhuma forma) a 10 (extremamente importante). A partir disso, o cliente seleciona, no máximo, 5 atividades para as quais atribui maior importância, e dá a cada uma delas uma pontuação, também numa escala de 1 a 10, em relação ao desempenho nessas atividades (1- não é capaz de desempenhar e 10- capaz de desempenhar muito bem) e à satisfação com o desempenho (1- não satisfeito de forma nenhuma e 10- extremamente satisfeito). Reunindose todos os escores de desempenho e de satisfação, são obtidas pontuações totais que poderão ser comparadas com os resultados de reavaliações para mensuração do progresso. Uma mudança na pontuação de 2 pontos ou mais é considerada, pelos autores da medida, como clinicamente significativa (CUP et al., 2003; CARSWELL, 2004). O tempo total para administração é de, em média, 30-45 minutos (WRESSLE et al., 2002; DEDDING et al., 2004).

A COPM é uma medida válida e confiável e tem sido utilizada de forma bem sucedida com diversos grupos de clientes, em diferentes locais (CARSWELL, 2004; EYSSEN et al., 2006; PARKER; SYKES, 2006; LEXELL et al., 2006; CHATFIELD; BECKETT, 2007). Os estudos atestam a versatilidade internacional e a aplicabilidade cultural da COPM, mesmo tendo ela sido desenvolvida em contexto cultural canadense (CARSWELL, 2004). A COPM foi publicada primeiramente em 1991, já tendo sido traduzida, até o ano de 2005, para 24 idiomas diferentes e utilizada por terapeutas ocupacionais em mais de 35 países ao redor do mundo (CARSWELL, 2004; McCOLL et al., 2005). Nos últimos anos, o número de publicações que utilizaram a COPM tem aumentado (CARSWELL, 2004). Recentemente, esse teste foi traduzido para o português e publicado no Brasil 
(LAW, 2009). Nesse artigo, a sigla original em inglês será mantida já que é utilizada internacionalmente como indexador na área de Terapia Ocupacional.

$\mathrm{Na}$ busca de consolidação e reconhecimento da profissão, a Terapia Ocupacional precisa adotar medidas de resultados que demonstrem a efetividade clínica e econômica de suas intervenções e facilitem a implementação da prática centrada no cliente. As pesquisas e a prática clínica indicam que a COPM pode ser útil nesse aspecto (CARSWELL, 2004).

O interesse desse estudo é realizar uma bibliografia comentada, analisando-se os artigos publicados nos últimos sete anos sobre a utilização da COPM no campo da saúde mental. Objetiva-se, assim, verificar a área de desempenho ocupacional na qual se concentram as dificuldades identificadas pelos clientes da saúde mental, bem como as vantagens e limitações da utilização do instrumento com esse público.

\section{METODOLOGIA}

A pesquisa bibliográfica foi feita através de consultas nas seguintes bases de dados eletrônicas: Medline, OTseeker e CINAHL. A língua inglesa foi utilizada como estratégia de busca, sendo essa restrita aos artigos publicados no período de 2002 a 2009. Os descritores utilizados foram "COPM" e "Canadian Ocupational Performance Measure".

A seleção dos artigos foi realizada por duas pesquisadoras de acordo com o seguinte critério de inclusão: artigos que contivessem, no título, as palavras-chave mental health e/ou psychiatric disorders e/ou schizophrenia associadas aos descritores COPM e/ou Canadian Occupational Performance Measure.

Os artigos selecionados foram analisados criticamente de acordo com a metodologia proposta por Law et al. (1998), que sugere a criação de um quadro de caracterização dos artigos, sendo esses resumidos de forma padronizada de acordo com os itens: autor(es); objetivo; desenho do estudo; amostra e resultados (Quadro 1).

\section{RESULTADOS}

Encontraram-se nas diferentes buscas seis artigos que atenderam aos critérios de inclusão. Desses, somente 4 estavam disponíveis em território nacional, sendo 1 eliminado pelo fato de não apresentar resultados da aplicação da COPM. $\mathrm{O}$ quadro que se segue ilustra a caracterização dos artigos (Quadro 1).

Quadro 1 - Caracterização dos artigos selecionados nesse trabalho, segundo metodologia proposta por Law et al. (1998).

\begin{tabular}{|c|c|c|c|c|}
\hline Autores & Objetivo do estudo & Desenho do & Amostra & Principais resultados \\
\hline $\begin{array}{l}\text { CRESSWELL, } \\
\text { M. K.; RUGG, } \\
\text { S. A. (2003) }\end{array}$ & $\begin{array}{l}\text { Abordar as seguintes questões: } \\
\text { 1. Quais são as mudanças re- } \\
\text { veladas pela COPM em um } \\
\text { grupo de indivíduos com es- } \\
\text { quizofrenia, seguindo-se } 12 \\
\text { semanas de intervenção de } \\
\text { Terapia Ocupacional? } \\
\text { 2. Os clientes identificam défi- } \\
\text { cits de desempenho ocupacional } \\
\text { em cada } 1 \text { das } 3 \text { categorias de } \\
\text { desempenho ocupacional? }\end{array}$ & $\begin{array}{l}\text { Quase- expe- } \\
\text { rimental do } \\
\text { tipo antes e } \\
\text { depois. }\end{array}$ & $\begin{array}{l}9 \text { participantes: } 5 \text { homens e } 4 \text { mulheres, } \\
\text { com idades entre } 17 \text { e } 60 \text { anos, com } \\
\text { média de } 32 \text { anos, e diagnóstico de } \\
\text { esquizofrenia, encaminhados a um ser- } \\
\text { viço de Terapia Ocupacional baseado na } \\
\text { comunidade. }\end{array}$ & $\begin{array}{l}\text { 1. As mudanças detectadas pela COPM encontradas tanto } \\
\text { nas pontuações de desempenho dos clientes quanto nas } \\
\text { de satisfação mostraram-se clinicamente significativas, } \\
\text { já que, em média, os resultados apontaram um aumento } \\
\text { de mais de } 2 \text { pontos. } \\
\text { 2. Os clientes foram capazes de identificar problemas } \\
\text { de desempenho ocupacional nas } 3 \text { áreas de desempenho } \\
\text { utilizando a COPM. } 40 \% \text { dos problemas referiam-se à } \\
\text { área de produtividade, } 35 \% \text { à de lazer e atividades sociais } \\
\text { e } 25 \% \text { à de auto-cuidado. }\end{array}$ \\
\hline $\begin{array}{l}\text { P A N, A.; } \\
\text { CHUNG, L.; } \\
\text { HSIN-HWEI, } \\
\text { G. (2003) }\end{array}$ & $\begin{array}{l}\text { Testar a confiabilidade e utili- } \\
\text { dade clínica da COPM em um } \\
\text { grupo de clientes com desor- } \\
\text { dens psiquiátricas em Taiwan } \\
\text { e descrever problemas relacio- } \\
\text { nados a atividades rotineiras } \\
\text { desses clientes. }\end{array}$ & $\begin{array}{l}\text { Metodológico } \\
\text { e descritivo. }\end{array}$ & $\begin{array}{l}\text { 141 participantes: } 82 \text { homens e } 59 \text { mu- } \\
\text { lheres, com idades entre } 17 \text { e } 62 \text { anos e } \\
\text { média de } 35,6 \text { anos. } 94 \% \text { dos sujeitos } \\
\text { apresentavam diagnóstico de esqui- } \\
\text { zofrenia. Os participantes provinham } \\
\text { de um instituto psiquiátrico e de um } \\
\text { hospital afiliado a uma universidade de } \\
\text { Taiwan, sendo atendidos em unidades } \\
\text { de tratamento destinadas ao paciente } \\
\text { com um quadro psiquiátrico agudo, } \\
\text { sub-agudo, crônico, de reabilitação e } \\
\text { psicossomático. }\end{array}$ & $\begin{array}{l}\text { O coeficiente de correlação intra-classe dos escores de } \\
\text { teste e reteste variaram de r }=0,842 \text { para desempenho a } \\
\mathrm{r}=0,847 \text { para satisfação com desempenho, o que demons- } \\
\text { tra uma confiabilidade satisfatória } \\
\text { Numa escala de } 1 \text { a } 7 \text {, o nível de dificuldade em quanti- } \\
\text { ficar a importância dos problemas da COPM foi de } 4,6 \text {, } \\
\text { para quantificar o desempenho, } 3,9 \text {, e para a satisfação } \\
\text { com o desempenho do cliente, também } 3,9 \text {. } \\
37 \% \text { dos problemas identificados referiam-se ao } \\
\text { auto-cuidado, } 25 \% \text { ao trabalho, } 20 \% \text { ao lazer e } 12 \% \\
\text { à socialização. }\end{array}$ \\
\hline $\begin{array}{l}\text { C H E S W O R- } \\
\text { TH, C. et al. } \\
(2002)\end{array}$ & $\begin{array}{l}\text { O estudo teve como objeti- } \\
\text { vos: estabelecer a utilidade da } \\
\text { COPM em medir mudanças em } \\
\text { clientes de saúde mental sub- } \\
\text { metidos à Terapia Ocupacional } \\
\text { e identificar no que consistiam } \\
\text { tais mudanças. }\end{array}$ & $\begin{array}{l}\text { Quase-experi- } \\
\text { mental do tipo } \\
\text { antes e depois }\end{array}$ & $\begin{array}{l}\text { 60 participantes: } 32 \text { mulheres e } 28 \\
\text { homens, com idades entre } 18 \text { e } 74 \text { anos } \\
\text { e média de } 46 \text { anos. Apresentavam } \\
\text { diversos problemas de saúde mental, } \\
\text { tais como ansiedade, depressão, falta de } \\
\text { segurança e condições psicóticas, e cons- } \\
\text { tituíam todos os clientes encaminhados } \\
\text { a uma unidade de Terapia Ocupacional } \\
\text { durante } 4 \text { anos. }\end{array}$ & $\begin{array}{l}\text { A diferença entre os níveis ao início e ao final da terapia, } \\
\text { tanto para desempenho, quanto para satisfação, mostrou- } \\
\text { se clinicamente significativa. } 68,3 \% \text { e } 71,7 \% \text { dos clientes } \\
\text { quantificaram, respectivamente, um aumento de } 2 \text { pontos } \\
\text { ou mais no desempenho e satisfação após intervenção } \\
\text { de Terapia Ocupacional. } \\
62,2 \% \text { dos problemas identificados enquadraram-se na } \\
\text { categoria de lazer, } 22,5 \% \text { na de produtividade e } 15,3 \% \\
\text { na de auto-cuidado. }\end{array}$ \\
\hline
\end{tabular}


BASTOS, S. C. A. et al. O uso da medida Canadense. Rev. Ter. Ocup. Univ. São Paulo, v. 21, n. 2, p. 104-110, maio/ago. 2010.

\section{DISCUSSÃO}

Os artigos selecionados apontam para o reconhecimento de uma dificuldade potencial para conceituar e medir níveis de funcionamento social em clientes de saúde mental (CRESSWELL; RUGG, 2003). Os autores argumentam que a clientela de saúde mental impõe alguns desafios que dificultam a utilização de instrumentação padronizada. Tais desafios podem incluir dificuldade de interação dos clientes, efeitos adversos consequentes ao uso de medicação, prejuízo na iniciativa e tomada de decisão, entre outros. A literatura alega ainda que há uma interação complexa entre fatores sociais, culturais e de saúde que também precisam ser levados em consideração quando se tenta documentar mudanças em um grupo de clientes (CHESWORTH et al., 2002). A necessidade de documentar o impacto das intervenções faz com que o uso de testes padronizados que fornecem informações quantitativas e qualitativas seja estimulado em Terapia Ocupacional. Cabe ressaltar que os resultados da avaliação decorrente do uso de instrumentação padronizada constitui-se como um dos elementos do processo terapêutico. Assim, torna-se necessário que a informação disponibilizada pelo teste seja analisada e interpretada no contexto do raciocínio clínico.

Considerando que o objetivo dessa pesquisa bibliográfica foi identificar a área de desempenho ocupacional na qual predominam dificuldades identificadas pelos clientes da saúde mental, constatou-se que o impacto não é específico em uma área, sugerindo que as condições de saúde na área de saúde mental repercutem em dificuldades de desempenho ocupacional no auto-cuidado, trabalho e lazer.

No estudo de Creswell e Rugg em 2003, conduzido com uma amostra de conveniência com clientes adultos encaminhados a um serviço de terapia ocupacional em saúde mental baseado na comunidade, constatou-se que a maior parte dos problemas identificados foi na área de desempenho da produtividade (40\%). As preocupações dos participantes relacionavam-se mais frequentemente ao trabalho, se protegido, voluntário ou pago. A segunda área de problemas de desempenho ocupacional eleita pelos clientes refere-se a ocupações de lazer ou sociais, confirmando o que a literatura registra sobre a dificuldade dos esquizofrênicos em continuar ou formar relações sociais (CRESSWELL; RUGG, 2003). Finalmente, esse estudo reflete a falta geral de preocupação dos clientes com problemas potenciais na área de auto-cuidado, diferentemente do constatado na pesquisa de Pan et al. (2003), cuja aplicação da COPM em pacientes psiquiátricos de Taiwan revelou que $37 \%$ dos problemas identificados se associavam a auto-cuidado, $25 \%$ a trabalho, $20 \%$ a atividades de lazer e $12 \%$ à socialização.
Na pesquisa feita por Chesworth et al. em 2002, os problemas quantificados pelos clientes revelaram maior frequência na área de lazer $(62,2 \%)$. Os autores alegam que talvez isso possa ser compreendido dado que clientes na saúde mental podem ter uma quantidade considerável de tempo disponível enquanto se submetem a programas terapêuticos. Isso traz uma questão interessante sobre a condução do tratamento de clientes nessa área porque muitos profissionais da saúde mental se focam na redução dos sintomas em detrimento da avaliação do funcionamento social e recreacional. Poder-se-ia afirmar que o uso desse instrumento identificou uma área de cuidado com o cliente que não é sempre reconhecida e priorizada terapeuticamente. Os autores sugerem que, talvez, os terapeutas ocupacionais pudessem reexaminar sua contribuição relativa a oportunidades de lazer para aqueles com dificuldades de saúde mental.

Os estudos que investigaram a aplicação da COPM em outros grupos clínicos detectaram, diferentemente da saúde mental, predomínio de dificuldades na área de autocuidado, como ilustra pesquisa com clientes da reabilitação física, em que $41 \%$ dos problemas se referiam à área mencionada (DEDDING et al., 2004). Resultado similar foi constatado em pesquisa com clientes acometidos por acidente vascular encefálico (CUP et al., 2003), com a constatação de que $45 \%$ dos problemas identificados se referiam ao auto-cuidado. Em trabalho com clientes de dois hospitais suecos das clínicas de geriatria, AVE e reabilitação em domicílio, também predominaram os problemas identificados na área de auto-cuidado (76\%) (WRESSLE et al., 2002). Em pesquisa com indivíduos com hemofilia (VAN MEETEREN et al., 2000), e em estudo com clientes com esclerose múltipla, foi detectado o auto-cuidado como a área de desempenho que concentrou mais dificuldades (51\%) (LEXELL et al., 2006).

Além das constatações referentes ao foco das queixas dos clientes relativas às áreas de desempenho ocupacional, os trabalhos que investigaram o uso da COPM na saúde mental trouxeram dados relevantes acerca da utilização deste instrumento no que diz respeito às limitações e vantagens associadas ao seu uso na clínica e na pesquisa.

\section{Limitações do instrumento}

Por meio dessa pesquisa bibliográfica foi possível evidenciar dificuldades relacionadas ao uso da COPM com clientes da saúde mental e de outros grupos clínicos. O fato de o cliente apresentar déficits cognitivos é assinalado como uma limitação de uso da medida, já que sua habilidade de tomar decisões fica restrita (CLEWES, 2003; PAN 
et al., 2003; CARSWELL, 2004; PARKER; SYKES, 2006; CHATFIELD; BECKETT, 2007). Os terapeutas ocupacionais que aplicaram a COPM ressaltaram ainda a dificuldade de classificar alguns dos problemas identificados pelos clientes nas áreas de desempenho ocupacional que o instrumento aborda (PAN et al., 2003). Os autores apontam também limitações decorrentes de problemas destacados pelos clientes que não podem ser resolvidos imediatamente, e da falta de concordância entre problemas citados pelo cliente e problemas identificados pelo terapeuta. Sobre esta última constatação, afirma-se que, a não ser quando se trata de questão que envolva a segurança do cliente, o terapeuta deve valorizar as prioridades do cliente, pois esse é o sujeito do processo terapêutico e suas reivindicações devem ser apreciadas pelo terapeuta (LAW, 2005). Salienta-se que esses aspectos caracterizam a prática centrada no cliente, a qual enfatiza a parceria entre cliente e profissional.

$O$ fato de o cliente não ter habilidade para identificar algum problema de desempenho ocupacional dificultou a aplicação do formulário (CHESWORTH et al., 2002; WARREN, 2002). Quando o cliente apresenta um quadro de sofrimento mental agudo, o uso da COPM pode fornecer informação imprecisa e, em alguns diagnósticos, tais como hipomania, depressão severa ou demência, podendo haver também dificuldades para obtenção de informação. Além disso, verificaram-se dificuldades quanto à pontuação, já que alguns clientes não estão aptos a compreender os números ou quantificar desempenho e satisfação. Com frequência, pacientes com sofrimento mental consideram difíceis os conceitos de auto-avaliação e sentem-se embaraçados para traduzir seus problemas em uma pontuação (WARREN, 2002; CHATFIELD; BECKETT, 2007; DEDDING et al., 2007).

\section{Vantagens do instrumento}

A COPM foi identificada como um instrumento útil para fornecer informação detalhada sobre o desempenho ocupacional do cliente (CRESSWELL; RUGG, 2003; DEDDING et al., 2007). Outro aspecto positivo diz respeito à possibilidade de desenvolver programas individualizados a partir da informação disponibilizada pelo instrumento e de detectar mudanças no desempenho, após um período de intervenção (CRESSWELL; RUGG, 2003; CARSWELL, 2004; LEXELL et al., 2006).

As pesquisas registram também que o uso da COPM, tanto na área de saúde mental quanto na saúde física, provê informação relevante para o planejamento do tratamento, permitindo a exploração de problemas de desempenho ocupacional que poderiam não ser detectados por meio de outros instrumentos padronizados (VAN MEETEREN et al., 2000; CUP et al., 2003; PAN et al., 2003; LEXELL et al., 2006; DEDDING et al., 2007). Foi constatado, ainda, que o envolvimento dos clientes no processo da COPM fez com que esses se tornassem mais motivados a colaborarem no planejamento de seu próprio tratamento (PAN et al., 2003).

Outra vantagem detectada por meio da aplicação da COPM diz respeito ao esclarecimento dos membros da equipe multidisciplinar sobre o papel do terapeuta ocupacional, repercutindo em encaminhamentos mais apropriados para essa modalidade de intervenção (WARREN, 2002; CRESSWELL; RUGG, 2003; CLEWES, 2003; CARSWELL, 2004; PARKER; SYKES, 2006). Tal vantagem pode contribuir para a minimização da incongruência entre a razão para se procurar o serviço, o procedimento de admissão, o serviço em si e seu resultado. Essa incongruência pode ter efeito negativo para a consolidação da identidade da profissão de Terapia Ocupacional (TROMBLY, 1993).

Os autores pesquisados salientam a importância de o terapeuta ocupacional ter conhecimento sobre o modelo da prática centrada no cliente e também ser treinado para utilizar o instrumento na prática clínica (WARREN, 2002; WRESSLE et al., 2002; CLEWES, 2003; PAN et al., 2003; PARKER; SYKES, 2006; CHATFIELD; BECKETT, 2007; DEDDING et al., 2007). A literatura aponta ainda que a experiência do terapeuta no campo da saúde mental é muito importante para a utilização do instrumento com o propósito de auxiliar os clientes no estabelecimento de objetivos realistas e na construção de planos de tratamento que visem à superação das dificuldades identificadas no desempenho ocupacional (CHESWORTH et al., 2002).

Essa pesquisa bibliográfica fez uso de uma literatura que é, em sua totalidade, internacional. Consequentemente, os resultados apresentados restringem-se a realidades sócioculturais distintas da realidade brasileira. Torna-se necessário que evidências sobre o uso da COPM em pacientes com sofrimento mental, no Brasil, sejam disponibilizadas, a fim de identificar quais são as dificuldades predominantes desse grupo clínico no que diz respeito às áreas de desempenho ocupacional. Mediante essas informações, o plano de tratamento do cliente poderá ser ajustado às suas demandas, beneficiando a recuperação funcional.

\section{CONCLUSÃO}

ACOPM é uma medida de resultados contemporânea, pois vai ao encontro dos valores que apoiam práticas de saúde atuais, que são centradas no cliente. Essa pesquisa bibliográfica revelou limitada literatura sobre a aplicação 
da COPM com pessoas que apresentam sofrimento mental, o que restringe a possibilidade de afirmar qual (is) a(s) dificuldade (s) de desempenho ocupacional mais freqüente(s) nesse grupo de clientes.

Convém destacar a singularidade das informações fornecidas pelo uso da COPM no que diz respeito ao desempenho ocupacional do cliente, bem como o (re) conhecimento que o uso dessa medida propicia sobre a atuação do terapeuta ocupacional na equipe multidisciplinar.
A despeito das dificuldades existentes na aplicação da COPM, que incluem o fato de o cliente apresentar déficit cognitivo ou quadro de sofrimento mental agudo, dificuldades referentes à pontuação, dentre outras, pode-se afirmar que esse instrumento foi identificado como uma ferramenta útil para uso na saúde mental. Compete aos terapeutas ocupacionais que lidam nesta área incorporarem em sua prática o uso rotineiro de instrumentos de avaliação padronizados, empreendendo esforços no exercício de divulgação dos resultados.

BASTOS, S. C. A.; MANCINI, M. C.; PYLÓ, R. M. The use of the canadian occupational performance measure in mental health. uso da medida canadense de desempenho ocupacional (COPM) em saúde mental. Rev. Ter. Ocup. Univ. São Paulo, v. 21, n. 2, p. 104-110, maio/ago. 2010.

\begin{abstract}
The COPM is an instrument capable of measuring the impact of an intervention for a human being, which has the purpose to detect changes in the perception of the client about his occupational performance along the time, as well as changes about his satisfaction in relation with his performance. A commentated bibliography was fulfilled, analyzing the published articles in the last seven years about the application of the COPM in the area of mental health. It was intended to examine the area of the occupational performance in which the identified difficulties by the clients of mental health are concentrated, as well as vantages and limitations of the application of this instrument with this public. The research was achieved through the electronic database Medline, OTseeker and CINAHL, during 2002-2009. The results suggest that the health conditions in the area of mental health reverberate into difficulties of occupational performance in the three areas. It's recommended to detach the singularity of the information given by the use of COPM when related to the occupational performance of the client, as well as the (re) knowledge that the use of this measure propitiate about the performance of the occupational therapist in the multidisciplinary team. Despite the existing difficulties in the application of the COPM, it can be affirmed that this instrument was identified as a useful tool to be used in mental health.
\end{abstract}

KEY WORDS: Health evaluation/methods; Indicators; Health human resource evaluation; Mental health; Occupational Therapy.

\section{REFERÊNCIAS}

SAMPAIO. R.; MANCINI, M. C.; FONSECA, S. T. Produção científica e atuação profissional: aspectos que limitam essa integração na Fisioterapia e Terapia Ocupacional. Rev. Bras. Fisioter., v. 6, n. 3, p. 113-118, 2002.

DEJONG , G.; SUTTON, J. Reab 2000: the evolution of medical rehabilitation in american health care. In: LANDRUM, P. K.; SCHIMIDT, N.; MCLEAN, A. Outcome-oriented rehabilitation. Principles, strategies and tools for effective program management. Gaithersburg, Maryland: Aspen Publishers, Inc. 1995. p.3-42.

POLlOCK, N.; McCOLL, M. A.; CARSWELL, A. Medida de performance ocupacional canadense. In: SUMSION, T.
Pratica baseada no cliente na terapia ocupacional: guia para implementacao. São Paulo: Roca, 2003.

EYSSEN, I.; BEELEN, A.; DEDDING, C.; CARDOL, M.; DEKKER, J. et al. The reproducibility of the canadian occupational performance measure. Clin. Rehabil., v. 19, p. 888-894, 2005. KIRKWOOD, R. Instrumentação. Aula proferida na UFMG, Belo Horizonte, 2006.

DEDDING, C.; CARDOL, M.; EYSSEN, I. C.; DEKKER, J.; BEELEN, A. et al. Validity of the canadian occupational performance measure: a client-centred outcome measurement. Clin. Rehabil., n. 18, p. 660-667, 2004. 
CUP, E.; SCHOLTE, O. P.; REIMER, W. J.; THIJSSEN, M. C.; VAN KUYK-MINIS, M. A. Realiability and validity of the canadian occupational performance measure in stroke patients. Clin. Rehabil., v. 17, p. 402-409, 2003.

CARSWELL, A. The Canadian occupational performance measure: a research and clinical literature review. Can. J. Occup. Ther., v. 71, n.4, p. 210-222, 2004.

WRESSlE, E.; EEG-OLOFSSON, A. M.; MARCUSSON, J.; HENRIKSSON, C. Improved client participation in the rehabilitation process using a client-centred goal formulation structure. J. Rehabil. Med., v. 34, p. 5-11, 2002.

PARKER, D.; SYKES, C. A Systematic review of the canadian occupational performance measure: a clinical practice perspective. Br. J. Occup. Ther., v. 69, n. 4, p. 150-160, 2006.

CHATFIELD, J.; BECKETT, D. The canadian occupational performance measure: use in an independent living centre. Int. J. Ther. Rehabil., v. 14, n. 6, p. 280-284, 2007.

LEXELL, E.; IWARSSON, S.; LEXELL, J. The complexity of daily occupations in multiple sclerosis. Scand. J. Occup. Ther., v. 13, p. 241-248, 2006.

McCOLL, M.; LAW, M.; BAPTISTE, S.; POLLOCK, N.; CARSWELL, A.; POLATAJKO, H. J. Targeted applications of the canadian occupational performance measure. Can. J. Occup. Ther., v. 72, n.5, p. 298-300, 2005.

LAW, M. Medida canadense de desempenho ocupacional (COPM). Org. Trad. de MAGALHÃES, L.; MAGALHÃES, L.; CARDOSO, A. Belo Horizonte: Editora UFMG, 2009.

CRESSWELL, M.; RUGG, S. The canadian performance occupational therapy measure: its use with clients with schizofrenia. Int. J. Ther. Rehabil., v. 10, n. 12, p. 544-553, 2003.

CHESWORTH, C.; DUFFY, R; HODNETT, J.; KNIGHT, A. Measuring clinical effectiveness in mental health: Is the Canadian Occupational Performance an appropriate measure? Br. J. Occup. Ther., v. 65, n. 1, p. 30-34, 2002.

VAN MEETEREN, N.; STRATO, I. H.; VAN VELDHOVEN, N. H.; DE KLEIJN, P.; VAN DEN BERG, H. M.; HELDERS, P. J. The utility of the dutch arthritis impact measurement scales 2 for assessing health status in individuals with haemophilia: a pilot study. Haemophilia, v. 6, 664-671, 2000.

PAN, A.; CHUNG, L.; HSIN-HWEI, G. Confiabilidade e validade da medida canadense de desempenho ocupacional para clientes com desordens psiquiátricas em Taiwan. Occup. Ther. Int., v. 10, n. 4, p. 269-277, 2003.

CLEWES, J. Reviewing the use of the canadianoccupational performance measure within the Manchester Mental Health Partnership. Mental Health Occup. Ther., v. 8, n. 1, p. 29-30, 2003.

LAW, M. COPM - Canadian occupational performance measure. Revisado em maio de 2005. Disponível em: http://www.caot.ca/ copm/index.htm. Acesso em: 29 out. 2008.

WARREN, A. An evaluation of the canadian model of occupational performance and the canadian occupational performance measure in mental health practice. Br. J. Occup. Ther., v. 65, n. 11, p. 515-521, 2002.

TROMBLY, C. Antecipando o futuro: avaliação da função ocupacional. Tradução de Júnia Jorge R. Cordeiro. Am. J. Occup. Ther., v. 47, n. 3, p. 253-257, 1993. 